\title{
Hardy type estimates for commutators of fractional integrals associated with Schrödinger operators
}

\section{Yinhong Xia, Min Chen*}

School of Mathematics and Statistics, Huanghuai University, Zhumadian 463000, P. R. China.

\author{
Communicated by $\mathrm{Y}$. Hu
}

\begin{abstract}
We consider the Schrödinger operator $\mathcal{L}=-\Delta+V$ on $\mathbb{R}^{n}$, where $n \geqslant 3$ and the nonnegative potential $V$ belongs to reverse Hölder class $\mathrm{RH}_{q_{1}}$ for some $\mathrm{q}_{1}>\frac{\mathrm{n}}{2}$. Let $\mathbb{I}_{\alpha}$ be the fractional integral associated with $\mathcal{L}$, and let $b$ belong to a new Campanato space $\Lambda_{\beta}^{\theta}(\rho)$. In this paper, we establish the boundedness of the commutators $\left[b, \mathbb{I}_{\alpha}\right]$ from $L^{p}\left(\mathbb{R}^{n}\right)$ to $L^{q}\left(\mathbb{R}^{n}\right)$ whenever $1 / q=1 / p-(\alpha+\beta) / n, 1<p<n /(\alpha+\beta)$. When $\frac{n}{n+\beta}<p \leqslant 1,1 / q=1 / p-(\alpha+\beta) / n$, we show that $\left[b, \mathbb{I}_{\alpha}\right]$ is bounded from $H_{\mathcal{L}}^{p}\left(\mathbb{R}^{n}\right)$ to $L^{q}\left(\mathbb{R}^{n}\right)$. Moreover, we also prove that $\left[b, \mathbb{I}_{\alpha}\right]$ maps $H_{\mathcal{L}}^{\frac{n}{n+\beta}}\left(\mathbb{R}^{n}\right)$ continuously into weak $L^{\frac{n}{n-\alpha}}\left(\mathbb{R}^{n}\right)$. (C)2017 All rights reserved.
\end{abstract}

Keywords: Schrödinger operator, commutator, Campanato space, fractional integral, Hardy space. 2010 MSC: 42B30, 35J10.

\section{Introduction and results}

Let $\mathcal{L}=-\Delta+V$ be a Schrödinger operator on $\mathbb{R}^{n}, n \geqslant 3$. The function $V$ is nonnegative, $V \neq 0$, and belongs to a reverse Hölder class $\mathrm{RH}_{\mathrm{q}_{1}}$ for some $\mathrm{q}_{1}>\frac{\mathrm{n}}{2}$, that is, there exists a constant $\mathrm{C}$ such that

$$
\left(\frac{1}{|B|} \int_{B} V(y)^{q_{1}} d y\right)^{1 / q_{1}} \leqslant \frac{C}{|B|} \int_{B} V(y) d y
$$

for every ball $\mathrm{B} \subset \mathbb{R}^{\mathrm{n}}$.

Suppose $V \in \mathrm{RH}_{\mathrm{q}_{1}}$ with $\mathrm{q}_{1}>\mathrm{n} / 2$. The fractional integral associated with $\mathcal{L}$ is defined by

$$
\mathbb{I}_{\alpha} f(x)=\mathcal{L}^{-\alpha / 2} f(x)=\int_{0}^{\infty} e^{-t \mathcal{L}}(f)(x) \frac{d t}{t^{-\alpha / 2+1}}
$$

for $0<\alpha<\mathrm{n}$. If $\mathcal{L}=-\Delta$ is the Laplacian on $\mathbb{R}^{\mathfrak{n}}$, then $\mathbb{I}_{\alpha}$ is the Riesz potential $\mathrm{I}_{\alpha}$, that is,

$$
I_{\alpha} f(x)=\int_{\mathbb{R}^{n}} \frac{f(y)}{|x-y|^{n-\alpha}} d y .
$$

\footnotetext{
*Corresponding author

Email addresses: xiayh03@163.com (Yinhong Xia), chenmin2002@yeah.net (Min Chen)
} 
As in [10], for a given potential $V \in \mathrm{RH}_{\mathrm{q}_{1}}$ with $\mathrm{q}_{1}>\mathrm{n} / 2$, we define the auxiliary function

$$
\rho(x)=\sup \left\{r>0: \frac{1}{r^{n-2}} \int_{B(x, r)} V(y) d y \leqslant 1\right\}, x \in \mathbb{R}^{n} .
$$

It is well-known that $0<\rho(x)<\infty$ for any $x \in \mathbb{R}^{n}$.

Let $\theta>0$ and $0<\beta<1$, according to [7], the new Campanato class $\Lambda_{\beta}^{\theta}(\rho)$ consists of the locally integrable functions $b$ such that

$$
\frac{1}{|B(x, r)|^{1+\beta / n}} \int_{B(x, r)}\left|b(y)-b_{B}\right| d y \leqslant C\left(1+\frac{r}{\rho(x)}\right)^{\theta}
$$

holds for all $x \in \mathbb{R}^{n}$ and $r>0$. A seminorm of $b \in \Lambda_{\beta}^{\theta}(\rho)$, denoted by $[b]_{\beta}^{\theta}$, is given by the infimum of the constants in the inequalities above.

Note that if $\theta=0, \Lambda_{\beta}^{\theta}(\rho)$ is the classical Campanato space. If $\beta=0, \Lambda_{\beta}^{\theta}(\rho)$ is exactly the space $\mathrm{BMO}_{\theta}(\rho)$ introduced in [1].

We recall the Hardy space associated with Schrödinger operator $\mathcal{L}$ which had been studied by Dziubański and Zienkiewicz in [4] and [5]. Because $V \in \mathrm{L}_{\text {loc }}^{\mathrm{q}_{1}}\left(\mathbb{R}^{\mathfrak{n}}\right)$, the Schrödinger operator $\mathcal{L}$ generates a $\left(\mathrm{C}_{0}\right)$ contraction semigroup $\left\{\mathrm{T}_{\mathrm{s}}^{\mathcal{L}}: \mathrm{s}>0\right\}=\left\{e^{-\mathrm{s} \mathcal{L}}: \mathrm{s}>0\right\}$. The maximal function associated with $\left\{T_{s}^{\mathcal{L}}: s>0\right\}$ is defined by $M^{\mathcal{L}} f(x)=\sup _{s>0}\left|T_{s}^{\mathcal{L}} f(x)\right|$. We always denote $\eta=2-n / q_{1}$ and $\delta^{\prime}=\min \{1, \eta\}$. For $\frac{n}{n+\delta^{\prime}}<p \leqslant 1$, the Hardy space $H_{\mathcal{L}}^{p}\left(\mathbb{R}^{n}\right)$ associated with Schrödinger operator $\mathcal{L}$ is defined as follows.

Definition 1.1. We say that $f$ is an element of $H_{\mathcal{L}}^{p}\left(\mathbb{R}^{\mathfrak{n}}\right)$ if the maximal function $M^{\mathcal{L}} f$ belongs to $L^{p}\left(\mathbb{R}^{\mathfrak{n}}\right)$. The quasi-norm of $f$ is defined by $\|f\|_{H_{\mathcal{L}}^{p}\left(\mathbb{R}^{n}\right)}=\left\|M^{\mathcal{L}} f\right\|_{L^{p}\left(\mathbb{R}^{n}\right)}$.

We introduce the concept of $\mathrm{H}_{\mathcal{L}}^{\mathrm{p}, \mathrm{q}}$-atom.

Definition 1.2. Let $\frac{n}{n+\delta^{\prime}}<p \leqslant 1 \leqslant q \leqslant \infty$. A function $a \in \mathrm{L}^{2}\left(\mathbb{R}^{n}\right)$ is called an $\mathrm{H}_{\mathcal{L}}^{\mathrm{p}, \mathrm{q}}$-atom if $\mathrm{r}<\rho\left(\mathrm{x}_{0}\right)$ and the following conditions hold:

(i) supp $a \subset B\left(x_{0}, r\right)$,

(ii) $\|\mathrm{a}\|_{\mathrm{Lq}_{\left(\mathbb{R}^{n}\right)}} \leqslant\left|\mathrm{B}\left(\mathrm{x}_{0}, \mathrm{r}\right)\right|^{1 / \mathrm{q}-1 / \mathrm{p}}$,

(iii) if $r<\rho\left(x_{0}\right) / 4$, then $\int_{B\left(x_{0}, r\right)} a(x) d x=0$.

We have the following atomic characterization of Hardy space.

Proposition 1.3 ([5]). Let $\frac{n}{n+\delta^{\prime}}<p \leqslant 1 \leqslant q \leqslant \infty$. Then $f \in H_{\mathcal{L}}^{p}\left(\mathbb{R}^{n}\right)$ if and only if $\mathrm{f}$ can be written as $f=\sum_{j} \lambda_{j} a_{j}$, where $a_{j}$ are $\mathrm{H}_{\mathcal{L}}^{p, q}$-atoms, $\sum_{j}\left|\lambda_{j}\right|^{p}<\infty$, and the sum converges in the $\mathrm{H}_{\mathcal{L}}^{p}\left(\mathbb{R}^{n}\right)$ quasi-norm. Moreover

$$
\|f\|_{H_{\mathcal{L}}^{\mathfrak{p}\left(\mathbb{R}^{n}\right)}} \approx \inf \left\{\left(\sum_{j}\left|\lambda_{j}\right|^{p}\right)^{1 / p}\right\},
$$

where the infimum is taken over all atomic decompositions of $\mathrm{f}$ into $\mathrm{H}_{\mathcal{L}}^{\mathrm{p}, \mathrm{q}}$-atoms.

The above atomic decomposition of $\mathrm{H}_{\mathcal{L}}^{\mathrm{p}}\left(\mathbb{R}^{\mathrm{n}}\right)$ implies that the Hardy space $\mathrm{H}_{\mathcal{L}}^{\mathrm{p}}\left(\mathbb{R}^{\mathrm{n}}\right)$ is larger than the classical Hardy space $H^{p}\left(\mathbb{R}^{\mathfrak{n}}\right)$. Especially, the Hardy space $\mathrm{H}_{\mathcal{L}}^{\mathfrak{p}}\left(\mathbb{R}^{\mathfrak{n}}\right)$ is exactly the local Hardy space $h^{p}\left(\mathbb{R}^{n}\right)$ introduced by Goldberg in [6] when the potential $V$ is a positive constant.

Let us consider the commutator associated with the Riesz potential $\mathrm{I}_{\alpha}$ and locally integrable function $b,\left[b, I_{\alpha}\right] f(x)=b(x) I_{\alpha} f(x)-I_{\alpha}(b f)(x)$. When $b \in B M O$, Chanillo proved in [3] that $\left[b, I_{\alpha}\right]$ is bounded from $L^{p}\left(\mathbb{R}^{n}\right)$ to $L^{q}\left(\mathbb{R}^{\mathfrak{n}}\right)$ with $1 / q=1 / p-\alpha / n, 1<p<n / \alpha$. When $b$ belongs to the Campanato space $\Lambda_{\beta}, 0<\beta<1$, Paluszyński in [9] showed that $\left[b, I_{\alpha}\right]$ is bounded from $L^{p}\left(\mathbb{R}^{n}\right)$ to $L^{q}\left(\mathbb{R}^{n}\right)$, where $1 / q=$ 
$1 / p-(\alpha+\beta) / n, 1<p<n /(\alpha+\beta)$. Furthermore, Lu et al. in [8] considered the boundedness of $\left[b, I_{\alpha}\right]$ on the classical Hardy spaces when $b \in \Lambda_{\beta}(0<\beta \leqslant 1)$. They proved that if $\frac{n}{n+\beta}<p \leqslant 1$ and $1 / q=$ $1 / p-(\alpha+\beta) / n,\left[b, I_{\alpha}\right]$ maps $H^{p}\left(\mathbb{R}^{n}\right)$ continuously into $L^{q}\left(\mathbb{R}^{n}\right)$. At the endpoint $p=\frac{n}{n+\beta}$, they also showed that $\left[b, I_{\alpha}\right]$ maps $H^{p}\left(\mathbb{R}^{n}\right)$ continuously into weak $L^{n /(n-\alpha)}\left(\mathbb{R}^{n}\right)$.

When $b \in \mathrm{BMO}_{\theta}(\rho)$, Bui in [2] obtained the boundedness of $\left[b, \mathbb{I}_{\alpha}\right]$ from $L^{p}\left(\mathbb{R}^{\mathfrak{n}}\right)$ to $L^{q}\left(\mathbb{R}^{\mathfrak{n}}\right)$ with $1 / \mathrm{q}=1 / \mathrm{p}-\alpha / \mathrm{n}, 1<\mathrm{p}<\mathrm{n} / \alpha$.

Inspired by the above results, in this paper, we are interested in the boundedness of $\left[b, \mathbb{I}_{\alpha}\right]$ when $b$ belongs to the new Campanato class $\Lambda_{\beta}^{\theta}(\rho)$. The results of this paper are as follows.

Theorem 1.4. Let $0<\alpha<\mathrm{n}$, and let $\mathrm{V} \in \mathrm{RH}_{\mathrm{q}_{1}}$ with $\mathrm{q}_{1}>\mathrm{n} / 2$. Then for any $\mathrm{b} \in \Lambda_{\beta}^{\theta}(\rho), 0<\beta<1$, the commutator $\left[b, \mathbb{I}_{\alpha}\right]$ is bounded from $\mathrm{L}^{\mathrm{p}}\left(\mathbb{R}^{\mathfrak{n}}\right)$ into $\mathrm{L}^{\mathrm{q}}\left(\mathbb{R}^{\mathrm{n}}\right)$, where $\frac{1}{\mathrm{q}}=\frac{1}{\mathrm{p}}-\frac{\alpha+\beta}{n}, 1<p<\frac{n}{\alpha+\beta}$.

Theorem 1.5. Let $0<\alpha<n$, and let $\mathrm{V} \in \mathrm{RH}_{\mathrm{q}_{1}}$ with $\mathrm{q}_{1}>\mathrm{n} / 2$. Suppose $\mathrm{b} \in \Lambda_{\beta}^{\theta}(\rho), 0<\beta<\delta^{\prime}$. If $\frac{\mathrm{n}}{\mathrm{n}+\beta}<\mathrm{p} \leqslant 1$ and $\frac{1}{\mathrm{q}}=\frac{1}{\mathrm{p}}-\frac{\alpha+\beta}{\mathrm{n}}$, then the commutator $\left[\mathrm{b}, \mathbb{I}_{\alpha}\right]$ is bounded from $\mathrm{H}_{\mathcal{L}}^{\mathrm{p}}\left(\mathbb{R}^{\mathrm{n}}\right)$ into $\mathrm{L}^{\mathrm{q}}\left(\mathbb{R}^{\mathrm{n}}\right)$.

Theorem 1.6. Let $0<\alpha<n$, and let $\mathrm{V} \in \mathrm{RH}_{\mathrm{q}_{1}}$ with $\mathrm{q}_{1}>\mathrm{n} / 2$. Suppose $\mathrm{b} \in \Lambda_{\beta}^{\theta}(\rho), 0<\beta<\delta^{\prime}$. Then the commutator $\left[\mathrm{b}, \mathbb{I}_{\alpha}\right]$ is bounded from $\mathrm{H}_{\mathcal{L}}^{\frac{n}{n+\beta}}\left(\mathbb{R}^{\mathfrak{n}}\right)$ into weak $\mathrm{L}^{\frac{n}{n-\alpha}}\left(\mathbb{R}^{\mathfrak{n}}\right)$.

Finally, we make some conventions on the notation. Throughout the whole paper, we always use $C$ to denote a positive constant, that is independent of the main parameters involved but whose value may differ from line to line. We shall use the symbol $A \lesssim B$ to indicate that there exists a constant $C$ such that $A \leqslant C B . A \approx B$ means that $A \lesssim B$ and $B \lesssim A$.

\section{Some preliminaries}

We would like to recall some important properties concerning the auxiliary function which will play an important role to obtain the main results.

Proposition 2.1 ([10]). Let $\mathrm{V} \in \mathrm{RH}_{\mathrm{n} / 2}$. For the auxiliary function $\rho$ there exist $\mathrm{C}$ and $\mathrm{k}_{0} \geqslant 1$ such that

$$
C^{-1} \rho(x)\left(1+\frac{|x-y|}{\rho(x)}\right)^{-k_{0}} \leqslant \rho(y) \leqslant C \rho(x)\left(1+\frac{|x-y|}{\rho(x)}\right)^{\frac{k_{0}}{1+k_{0}}}
$$

for all $x, y \in \mathbb{R}^{n}$.

A ball $B(x, \rho(x))$ is called critical. Assume that $Q=B\left(x_{0}, \rho\left(x_{0}\right)\right)$, for $x \in Q$, the inequality above tells us that $\rho(x) \approx \rho(y)$ if $|x-y|<C \rho(x)$.

It is easy to get the following result from Proposition 2.1.

Lemma 2.2. Let $\mathrm{k} \in \mathbb{N}$ and $x \in 2^{\mathrm{k}+1} \mathrm{~B}\left(\mathrm{x}_{0}, \mathrm{r}\right) \backslash 2^{\mathrm{k}} \mathrm{B}\left(\mathrm{x}_{0}, \mathrm{r}\right)$. Then we have

$$
\frac{1}{\left(1+\frac{2^{k} r}{\rho(x)}\right)^{N}} \lesssim \frac{1}{\left(1+\frac{2^{k} r}{\rho\left(x_{0}\right)}\right)^{N /\left(k_{0}+1\right)}}
$$

Proposition 2.3 ([4]). There exists a sequence of points $\left\{x_{k}\right\}_{k=1}^{\infty}$ in $\mathbb{R}^{n}$, so that the family of critical balls $Q_{k}=$ $B\left(x_{k}, \rho\left(x_{k}\right)\right), k \geqslant 1$, satisfies

(i) $\bigcup_{k} Q_{k}=\mathbb{R}^{n}$;

(ii) there exists $N=N(\rho)$ such that for every $k \in N$, $\operatorname{card}\left\{j: 4 Q_{j} \cap 4 Q_{k}\right\} \leqslant N$.

Given $\alpha>0$, we define the following maximal functions for $g \in \mathrm{L}_{\mathrm{loc}}^{1}\left(\mathbb{R}^{\mathrm{n}}\right)$ and $x \in \mathbb{R}^{\mathrm{n}}$,

$$
\begin{aligned}
& M_{\rho, \alpha} g(x)=\sup _{x \in B \in \mathcal{B}_{\rho, \alpha}} \frac{1}{|B|} \int_{B}|g(y)| d y, \\
& M_{\rho, \alpha}^{\sharp} g(x)=\sup _{x \in B \in \mathcal{B}_{\rho, \alpha}} \frac{1}{|B|} \int_{B}\left|g(y)-g_{B}\right| d y,
\end{aligned}
$$

where $\mathcal{B}_{\rho, \alpha}=\left\{B(z, r): z \in \mathbb{R}^{n}\right.$ and $\left.r \leqslant \alpha \rho(y)\right\}$. 
We have the following Fefferman-Stein type inequality.

Proposition 2.4 ([1]). For $1<p<\infty$, there exist $\eta$ and $\gamma$ such that if $\left\{Q_{k}\right\}_{k}$ is a sequence of balls as in Proposition 2.3 , then

$$
\int_{\mathbb{R}^{n}}\left|M_{\rho, \eta} g(x)\right|^{p} \mathrm{~d} x \lesssim \int_{\mathbb{R}^{n}}\left|M_{\rho, \gamma}^{\sharp} \mathrm{g}(x)\right|^{p} \mathrm{~d} x+\sum_{k}\left|Q_{k}\right|\left(\frac{1}{\left|Q_{k}\right|} \int_{2 Q_{k}}|g|\right)^{p}
$$

for all $\mathrm{g} \in \mathrm{L}_{\mathrm{loc}}^{1}\left(\mathbb{R}^{\mathrm{n}}\right)$.

Let us recall the property of $b \in \Lambda_{\beta}^{\theta}(\rho)$.

Lemma 2.5 ([7]). Let $1 \leqslant s<\infty, b \in \Lambda_{\beta}^{\theta}(\rho)$, and $B=B(x, r)$. Then

$$
\left(\frac{1}{\left|2^{k} B\right|} \int_{2^{k} B}\left|b(y)-b_{B}\right|^{s} d y\right)^{1 / s} \leqslant[b]_{\beta}^{\theta}\left(2^{k} r\right)^{\beta}\left(1+\frac{2^{k} r}{\rho(x)}\right)^{\theta^{\prime}}
$$

for all $\mathrm{k} \in \mathbb{N}$, where $\theta^{\prime}=\left(\mathrm{k}_{0}+1\right) \theta$, and $\mathrm{k}_{0}$ is the constant appearing in Proposition 2.1.

Proposition 2.6 ([5]). Let $\mathrm{p}_{\mathrm{t}}(\mathrm{x}, \mathrm{y})$ be the kernels associated with the semigroups $\left\{\mathrm{e}^{-\mathrm{t} \mathcal{L}}\right\}_{\mathrm{t}>0}$. If $\mathrm{V} \in \mathrm{RH}_{\mathrm{q}_{1}}$ with $\mathrm{q}_{1}>\mathrm{n} / 2$, then for every $0<\delta<\delta^{\prime}$ and every $\mathrm{N}>0$ there exists a constant $\mathrm{C}>0$ such that for $|\mathrm{y}-z|<\frac{1}{2}|x-y|$, we have

$$
\left|p_{t}(x, y)-p_{t}(x, z)\right|+\left|p_{t}(y, x)-p_{t}(z, x)\right| \lesssim \frac{1}{t^{n / 2}}\left(\frac{|y-z|}{\sqrt{t}}\right)^{\delta} \exp \left(-\frac{|x-y|^{2}}{5 t}\right)\left(1+\frac{\sqrt{t}}{\rho(x)}+\frac{\sqrt{t}}{\rho(y)}\right) .
$$

Let $K_{\alpha}$ be the kernel of $\mathbb{I}_{\alpha}$. The following results give the estimates on the kernel $K_{\alpha}(x, y)$.

Lemma 2.7. Suppose $\mathrm{V} \in \mathrm{RH}_{\mathrm{q}_{1}}$ with $\mathrm{q}_{1}>\frac{\mathrm{n}}{2}$.

(i) For every $\mathrm{N}>0$, there exists a constant $\mathrm{C}$ such that

$$
\left|K_{\alpha}(x, y)\right| \lesssim \frac{1}{\left(1+\frac{|x-y|}{\rho(x)}\right)^{N}} \frac{1}{|x-y|^{n-\alpha}} .
$$

(ii) For every $0<\delta<\delta^{\prime}$ there exists a constant $\mathrm{C}$ such that for every $\mathrm{N}>0$, we have

$$
\left|\mathrm{K}_{\alpha}(x, y)-\mathrm{K}_{\alpha}(x, z)\right|+\left|\mathrm{K}_{\alpha}(\mathrm{y}, \mathrm{x})-\mathrm{K}_{\alpha}(z, x)\right| \lesssim \frac{1}{\left(1+\frac{|x-y|}{\rho(x)}\right)^{\mathrm{N}}} \frac{|y-z|^{\delta}}{|x-y|^{\mathrm{n}+\delta-\alpha}}
$$

where $|y-z| \leqslant|x-y| / 4$.

Proof. We observe that (i) is the result of Proposition 3.3 of [2]. By Proposition 2.4 and the methods used in Proposition 3.3 of [2], we can obtain (ii).

Since $\left|\mathbb{I}_{\alpha}(f)(x)\right| \lesssim I_{\alpha}(|f|)(x)$, then we get the following.

Corollary 2.8. Suppose $\mathrm{V} \in \mathrm{RH}_{\mathrm{q}_{1}}$ with $\mathrm{q}_{1}>\mathrm{n} / 2$. Let $0<\alpha<\mathrm{n}$ and let $1 \leqslant \mathrm{p}<\mathrm{q}<\infty$ satisfy $1 / \mathrm{q}=$ $1 / p-\alpha / n$. Then for all $f$ in $\mathrm{L}^{p}\left(\mathbb{R}^{\mathfrak{n}}\right)$ we have

$$
\left\|\mathbb{I}_{\alpha} f\right\|_{L^{q}\left(\mathbb{R}^{n}\right)} \lesssim\|f\|_{L^{p}\left(\mathbb{R}^{n}\right)}
$$

when $\mathrm{p}>1$, and also

$$
\left\|\mathbb{I}_{\alpha} f\right\|_{W L q\left(\mathbb{R}^{n}\right)} \lesssim\|f\|_{L^{1}\left(\mathbb{R}^{n}\right)}
$$

when $p=1$. 


\section{Proof of Theorem 1.4}

In order to prove Theorem 1.4, we need the following lemmas.

Lemma 3.1. Let $1<s<p<n /(\alpha+\beta), b \in \Lambda_{\beta}^{\theta}(\rho)$, and $Q=B\left(x_{0}, \rho\left(x_{0}\right)\right)$. Then

$$
\frac{1}{|Q|} \int_{Q}\left|\left[b, \mathbb{I}_{\alpha}\right] f(y)\right| d y \lesssim[b]_{\beta}^{\Theta} \inf _{\chi \in Q} M_{\alpha+\beta, s}(f)(x),
$$

where

$$
M_{\alpha+\beta, s}(f)(x)=\sup _{x \in B}\left(\frac{1}{|B|^{1-(\alpha+\beta) s / n}} \int_{B}|f(y)|^{s} d y\right)^{1 / s} .
$$

Proof. Since

$$
\left[b, \mathbb{I}_{\alpha}\right] f(y)=\left(b(y)-b_{Q}\right) \mathbb{I}_{\alpha} f(y)-\mathbb{I}_{\alpha}\left(\left(b-b_{Q}\right) f\right)(y),
$$

we have

$$
\frac{1}{|Q|} \int_{Q}\left|\left[b, \mathbb{I}_{\alpha}\right] f(y)\right| d y \leqslant \frac{1}{|Q|} \int_{Q}\left|\left(b(y)-b_{Q}\right) \mathbb{I}_{\alpha} f(y)\right| d y+\frac{1}{|Q|} \int_{Q}\left|\mathbb{I}_{\alpha}\left(\left(b-b_{Q}\right) f\right)(y)\right| d y=I_{1}+I_{2} .
$$

By Hölder's inequality and Lemma 2.5 , for any $t>1$ we get

$$
\begin{aligned}
\mathrm{I}_{1} & \leqslant\left(\frac{1}{|\mathrm{Q}|} \int_{\mathrm{Q}}\left|\mathrm{b}(\mathrm{y})-\mathrm{b}_{\mathrm{Q}}\right|^{\mathrm{t}^{\prime}} \mathrm{d} y\right)^{1 / \mathrm{t}^{\prime}}\left(\frac{1}{|\mathrm{Q}|} \int_{\mathrm{Q}}\left|\mathbb{I}_{\alpha} \mathrm{f}(\mathrm{y})\right|^{\mathrm{t}} \mathrm{d} y\right)^{1 / \mathrm{t}} \\
& \lesssim[\mathrm{b}]_{\beta}^{\theta} \rho\left(x_{0}\right)^{\beta}\left(\left(\frac{1}{|\mathrm{Q}|} \int_{\mathrm{Q}}\left|\mathbb{I}_{\alpha} \mathrm{f}_{1}(\mathrm{y})\right|^{\mathrm{t}} \mathrm{d} y\right)^{1 / \mathrm{t}}+\left(\frac{1}{|\mathrm{Q}|} \int_{\mathrm{Q}}\left|\mathbb{I}_{\alpha} \mathrm{f}_{2}(\mathrm{y})\right|^{\mathrm{t}} \mathrm{d} y\right)^{1 / \mathrm{t}}\right)=\mathrm{I}_{11}+\mathrm{I}_{12}
\end{aligned}
$$

where $f=f_{1}+f_{2}$ with $f_{1}=f \chi_{2} Q$. Choose $t>1$ such that $1 / s-1 / t=\alpha / n$, then by the $\left(L^{s}, L^{t}\right)-$ boundedness of $\mathbb{I}_{\alpha}$ (see Corollary 2.8), we have

$$
\begin{aligned}
I_{11} & \lesssim[b]_{\beta}^{\Theta} \rho\left(x_{0}\right)^{\beta} \frac{1}{|Q|^{1 / t}}\left(\int_{2 Q}|f(y)|^{s} d y\right)^{1 / s} \\
& \lesssim[b]_{\beta}^{\Theta}\left(\frac{1}{|2 Q|^{1-(\alpha+\beta) s / n}} \int_{2 Q}|f(y)|^{s} d y\right)^{1 / s} \lesssim[b]_{\beta}^{\Theta} \inf _{x \in Q} M_{\alpha+\beta, s}(f)(x) .
\end{aligned}
$$

Note that

$$
\left|\mathbb{I}_{\alpha} \mathrm{f}_{2}(\mathrm{y})\right| \leqslant \int_{(2 \mathrm{Q})^{\mathrm{c}}}\left|\mathrm{K}_{\alpha}(\mathrm{y}, z) \mathrm{f}(z)\right| \mathrm{d} z \lesssim \int_{(2 \mathrm{Q})^{\mathrm{c}}} \frac{|\mathrm{f}(z)|}{\left(1+\frac{|y-z|}{\rho(y)}\right)^{\mathrm{N}}|\mathrm{y}-z|^{\mathrm{n}-\alpha}} \mathrm{d} z .
$$

In this situation, we have $\rho(y) \approx \rho\left(x_{0}\right),|y-z| \approx\left|x_{0}-z\right|$ for any $y \in Q$ and $z \in(2 Q)^{c}$. So, decomposing $(2 \mathrm{Q})^{\mathrm{c}}$ into annuli $2^{\mathrm{k}} \mathrm{Q} \backslash 2^{\mathrm{k}-1} \mathrm{Q}, \mathrm{k} \geqslant 2$, we get

$$
\left|\mathbb{I}_{\alpha} f_{2}(y)\right| \lesssim \sum_{k \geqslant 2} \frac{2^{-k N}}{\left|2^{k} Q\right|^{1-\alpha / n}} \int_{2^{k} Q}|f(z)| d z .
$$

Then, by Hölder's inequality we get

$$
\mathrm{I}_{12} \lesssim[\mathrm{b}]_{\beta}^{\Theta} \rho\left(x_{0}\right)^{\beta} \sum_{k \geqslant 2} \frac{2^{-k N}}{\left|2^{k} \mathrm{Q}\right|^{1-\alpha / n}} \int_{2^{k} \mathrm{Q}}|\mathrm{f}(z)| \mathrm{d} z
$$




$$
\begin{aligned}
& \lesssim[b]_{\beta}^{\Theta} \sum_{k \geqslant 2} \frac{2^{-k N}}{\left|2^{k} Q\right|^{1-(\alpha+\beta) / n}} \int_{2^{k} Q}|f(z)| d z \\
& \lesssim[b]_{\beta}^{\theta} \sum_{k \geqslant 2} 2^{-k N}\left(\frac{1}{\left|2^{k} Q\right|^{1-(\alpha+\beta) s / n}} \int_{2^{k} Q}|f(z)|^{s} d z\right)^{1 / s} \\
& \lesssim[b]_{\beta}^{\Theta} \inf _{x \in Q} M_{\alpha+\beta, s}(f)(x) .
\end{aligned}
$$

The estimate for $I_{2}$ can be proceeded in the same way of $I_{1}$. The decomposition $f=f_{1}+f_{2}$ gives

$$
\mathrm{I}_{2} \leqslant \frac{1}{|\mathrm{Q}|} \int_{\mathrm{Q}}\left|\mathbb{I}_{\alpha}\left(\left(b-\mathrm{b}_{\mathrm{Q}}\right) \mathrm{f}_{1}\right)(\mathrm{y})\right| \mathrm{d} y+\frac{1}{|\mathrm{Q}|} \int_{\mathrm{Q}}\left|\mathbb{I}_{\alpha}\left(\left(\mathrm{b}-\mathrm{b}_{\mathrm{Q}}\right) \mathrm{f}_{2}\right)(\mathrm{y})\right| \mathrm{d} y=\mathrm{I}_{21}+\mathrm{I}_{22} \text {. }
$$

Choose $r$ such that $1<\mathrm{r}<\mathrm{s}<\mathrm{p}$ and $1 / \mathrm{r}-1 / \mathrm{r}_{0}=\alpha / \mathrm{n}$. By Hölder's inequality, Lemma 2.5 and $\left(\mathrm{L}^{\mathrm{r}}, \mathrm{L}^{\mathrm{r}_{0}}\right)-$ boundedness of $\mathbb{I}_{\alpha}$, for some $u>r$ we have

$$
\begin{aligned}
\mathrm{I}_{21} & \lesssim\left(\frac{1}{|\mathrm{Q}|} \int_{\mathrm{Q}}\left|\mathbb{I}_{\alpha}\left(\left(\mathrm{b}-\mathrm{b}_{\mathrm{Q}}\right) \mathrm{f}_{1}\right)(\mathrm{y})\right|^{\mathrm{r}_{0}} \mathrm{~d} y\right)^{1 / \mathrm{r}_{0}} \\
& \lesssim \frac{1}{|\mathrm{Q}|^{-\alpha / n}}\left(\frac{1}{|\mathrm{Q}|} \int_{2 \mathrm{Q}}\left|\left(\left(\mathrm{b}-\mathrm{b}_{\mathrm{Q}}\right) \mathrm{f}_{1}\right)(\mathrm{y})\right|^{\mathrm{r}} \mathrm{d} y\right)^{1 / \mathrm{r}} \\
& \lesssim \frac{1}{|\mathrm{Q}|^{-\alpha / n}}\left(\frac{1}{|\mathrm{Q}|} \int_{2 \mathrm{Q}}|\mathrm{f}(\mathrm{y})|^{\mathrm{s}} \mathrm{d} y\right)^{1 / \mathrm{s}}\left(\frac{1}{|\mathrm{Q}|} \int_{2 \mathrm{Q}}\left|\mathrm{b}(\mathrm{y})-\mathrm{b}_{\mathrm{Q}}\right|^{\mathrm{u}} \mathrm{d} y\right)^{1 / \mathrm{u}} \\
& \lesssim[\mathrm{b}]_{\beta}^{\theta} \inf _{\mathrm{x} \in \mathrm{Q}} \mathrm{M}_{\alpha+\beta, s}(\mathrm{f})(\mathrm{x}) .
\end{aligned}
$$

The estimate $I_{22} \lesssim[b]_{\beta}^{\theta} \inf _{x \in Q} M_{\alpha+\beta, s}(f)(x)$ can be obtained by the similar approach to ones of $I_{12}$ and $I_{21}$. Then we omit the details here.

Lemma 3.2. Let $\mathrm{B}=\mathrm{B}\left(\mathrm{x}_{0}, \mathrm{r}\right)$ with $\mathrm{r} \leqslant \gamma \rho\left(\mathrm{x}_{0}\right)$ and let $\mathrm{x} \in \mathrm{B}$, then for any $\mathrm{y}, z \in \mathrm{B}$ we have

$$
\int_{(2 B) c}\left|K_{\alpha}(y, u)-K_{\alpha}(z, u)\right|\left|b(u)-b_{B} \| f(u)\right| d u \lesssim[b]_{\beta}^{\theta} M_{\alpha+\beta, s}(f)(x) .
$$

Proof. Setting $Q=B\left(x_{0}, \gamma \rho\left(x_{0}\right)\right)$, due to the facts that $\rho(y) \approx \rho(z) \approx \rho\left(x_{0}\right)$ and $|y-u| \approx|z-u| \approx\left|x_{0}-u\right|$, then by Lemma 2.7 we get

$$
\int_{(2 B)^{c}}\left|K_{\alpha}(y, u)-K_{\alpha}(z, u)\left\|b(u)-b_{B}\right\| f(u)\right| d u \lesssim K_{1}+K_{2},
$$

where

$$
K_{1}=r^{\delta} \int_{Q \backslash 2 B} \frac{\left|f(u)\left(b(u)-b_{B}\right)\right|}{\left|x_{0}-u\right|^{n+\delta-\alpha}} d u
$$

and

$$
K_{2}=r^{\delta} \rho\left(x_{0}\right)^{N} \int_{Q^{c}} \frac{\left|f(u)\left(b(u)-b_{B}\right)\right|}{\left|x_{0}-u\right|^{n+N+\delta-\alpha}} d u
$$

Let $j_{0}$ be the least integer such that $2^{j_{0}} \geqslant \gamma \rho\left(x_{0}\right) / r$. Splitting into annuli, we have

$$
\mathrm{K}_{1} \leqslant \sum_{j=2}^{j_{0}} 2^{-\delta j}\left(2^{j} r\right)^{\alpha} \frac{1}{\left|2^{j} B\right|} \int_{2^{j} B}|f(u)|\left|b(u)-b_{B}\right| d u .
$$


By Hölder's inequality, Lemma 2.5 and $2^{j} r \leqslant \gamma \rho\left(x_{0}\right)$ for $j<j_{0}$, we have

$$
\begin{aligned}
K_{1} & \lesssim \sum_{j=2}^{j_{0}} 2^{-j \delta}\left(2^{j} r\right)^{\alpha}\left(\frac{1}{\left|2^{j} B\right|} \int_{2^{j} B}|f(u)|^{s} d u\right)^{1 / s}\left(\frac{1}{\left|2^{j} B\right|} \int_{2^{j} B}\left|b(u)-b_{B}\right|^{s^{\prime}} d u\right)^{1 / s^{\prime}} \\
& \lesssim[b]_{\beta}^{\Theta} \sum_{j=2}^{j_{0}} 2^{-\delta j}\left(2^{j} r\right)^{\alpha+\beta}\left(1+\frac{2^{j} r}{\rho\left(x_{0}\right)}\right)^{\theta^{\prime}}\left(\frac{1}{\left|2^{j} B\right|} \int_{2^{j} B}|f(u)|^{s} d u\right)^{1 / s} \\
& \lesssim[b]_{\beta}^{\theta} M_{\alpha+\beta, s}(f)(x) .
\end{aligned}
$$

Note that

$$
\frac{1}{\left|2^{j} B\right|} \int_{2^{j} B}\left|f(u) \| b(u)-b_{B}\right| d u \lesssim[b]_{\beta}^{\theta}\left(2^{j} r\right)^{\beta}\left(1+\frac{2^{j} r}{\rho\left(x_{0}\right)}\right)^{\theta^{\prime}}\left(\frac{1}{\left|2^{j} B\right|} \int_{2^{j} B}|f(u)|^{s} d u\right)^{1 / s} .
$$

Since $\frac{2^{j} r}{\rho\left(x_{0}\right)} \geqslant \gamma$ for $j \geqslant j_{0}$, then, by choosing $N>\theta^{\prime}$ we get

$$
\begin{aligned}
K_{2} & \lesssim \rho\left(x_{0}\right)^{N} \sum_{j \geqslant j_{0}} 2^{-j \delta} \frac{1}{\left(2^{j} r\right)^{N-\alpha}} \frac{1}{\left|2^{j} B\right|} \int_{2^{j} B}|f(u)|\left|b(u)-b_{B}\right| d u \\
& \lesssim[b]_{\beta}^{\theta}\left(\frac{2^{j} r}{\rho\left(x_{0}\right)}\right)^{-\left(N-\theta^{\prime}\right)} \sum_{j=j_{0}}^{\infty} 2^{-j \delta}\left(2^{j} r\right)^{\alpha+\beta}\left(\frac{1}{\left|2^{j} B\right|} \int_{2^{j} B}|f(u)|^{s} d u\right)^{1 / s} \\
& \lesssim[b]_{\beta}^{\theta} M_{\alpha+\beta, s} f(x) .
\end{aligned}
$$

Lemma 3.3. Let $1<s<p<n /(\alpha+\beta), B=B\left(x_{0}, r\right)$ with $r \leqslant \gamma \rho\left(x_{0}\right)$, and $x \in B$. Then

$$
M_{\rho, \gamma}^{\sharp}\left(\left[b, \mathbb{I}_{\alpha}\right] f\right)(x) \lesssim[b]_{\beta}^{\Theta}\left(M_{\alpha+\beta, s}(f)(x)+M_{\beta, s}\left(\mathbb{I}_{\alpha} f\right)(x)\right) .
$$

Proof. We write

$$
\begin{aligned}
\frac{1}{|B|} \int_{B}\left|\left[b, \mathbb{I}_{\alpha}\right] f(y)-\left(\left[b, \mathbb{I}_{\alpha}\right] f\right)_{B}\right| d y \leqslant & \frac{2}{|B|} \int_{B}\left|\left(b(y)-b_{B}\right) \mathbb{I}_{\alpha} f(y)\right| d y+\frac{2}{|B|} \int_{B}\left|\mathbb{I}_{\alpha}\left(\left(b-b_{B}\right) f_{1}\right)(y)\right| d y \\
& +\frac{1}{|B|} \int_{B}\left|\mathbb{I}_{\alpha}\left(\left(b-b_{B}\right) f_{2}\right)(y)-\left(\mathbb{I}_{\alpha}\left(\left(b-b_{B}\right) f_{2}\right)\right)_{B}\right| d y \\
= & J_{1}+J_{2}+J_{3}
\end{aligned}
$$

where $f=f_{1}+f_{2}$ with $f_{1}=f \chi_{2 B}$.

Since $r \leqslant \gamma \rho\left(x_{0}\right)$ and $\rho(x) \approx \rho\left(x_{0}\right)$, by Hölder's inequality and Lemma 2.5, we get

$$
\begin{aligned}
J_{1} & \leqslant\left(\frac{1}{|B|} \int_{B}\left|b(y)-b_{B}\right|^{s^{\prime}} d y\right)^{1 / s^{\prime}}\left(\frac{1}{|B|} \int_{B}\left|\mathbb{I}_{\alpha} f(y)\right|^{s} d y\right)^{1 / s} \\
& \lesssim[b]_{\beta}^{\Theta} r^{\beta}\left(\frac{1}{|B|} \int_{B}\left|\mathbb{I}_{\alpha} f(y)\right|^{s} d y\right)^{1 / s} \lesssim[b]_{\beta}^{\theta} M_{\beta, s}\left(\mathbb{I}_{\alpha} f\right)(x) .
\end{aligned}
$$

For some $1<\mathrm{r}<\mathrm{s}$, and $1 / \mathrm{r}-1 / \mathrm{r}_{0}=\alpha / \mathrm{n}$, by Hölder's inequality and Lemma 2.5 , we have

$$
\begin{aligned}
\mathrm{J}_{2} & \lesssim\left(\frac{1}{|\mathrm{~B}|} \int_{\mathrm{B}}\left|\mathrm{I}_{\alpha}\left(\left(\mathrm{b}-\mathrm{b}_{\mathrm{B}}\right) \mathrm{f}_{1}\right)(\mathrm{y})\right|^{\mathrm{r}_{0}} \mathrm{dy}\right)^{1 / \mathrm{r}_{0}} \\
& \lesssim \frac{1}{|\mathrm{~B}|^{-\alpha / n}}\left(\frac{1}{|\mathrm{~B}|} \int_{2 \mathrm{~B}}\left|\left(\mathrm{~b}(\mathrm{y})-\mathrm{b}_{\mathrm{B}}\right) f(y)\right|^{\mathrm{r}} \mathrm{d} y\right)^{1 / \mathrm{r}}
\end{aligned}
$$




$$
\begin{aligned}
& \lesssim \frac{1}{|B|^{-\alpha / n}}\left(\frac{1}{|B|} \int_{2 B}\left|b(y)-b_{B}\right|^{u} d y\right)^{1 / u}\left(\frac{1}{|B|} \int_{2 B}|f(y)|^{s} d y\right)^{1 / s} \\
& \lesssim[b]_{\beta}^{\Theta}\left(\frac{1}{|2 B|^{1-(\alpha+\beta) s / n}} \int_{2 B}|f(y)|^{s} d y\right)^{1 / s} \\
& \lesssim[b]_{\beta}^{\theta} M_{\alpha+\beta, s}(f)(x) .
\end{aligned}
$$

By Lemma 3.2,

$$
\begin{aligned}
J_{3} & \leqslant \frac{1}{|B|^{2}} \int_{B} \int_{B} \int_{(2 B)^{c}}\left|K_{\alpha}(y, u)-K_{\alpha}(z, u)\left\|b(u)-b_{B}\right\| f(u)\right| d u d z d y \\
& \lesssim \int_{(2 B)^{c}}\left|K_{\alpha}(y, u)-K_{\alpha}(z, u)\left\|b(u)-b_{B}\right\| f(u)\right| d u \\
& \lesssim[b]_{\beta}^{\theta} M_{\alpha+\beta, s}(f)(x) .
\end{aligned}
$$

We now come to prove Theorem 1.4. By proposition 2.4, Lemma 3.1, and Lemma 3.3 we have

$$
\begin{aligned}
\left\|\left[b, \mathbb{I}_{\alpha}\right] f\right\|_{L^{q}\left(\mathbb{R}^{n}\right)}^{q} & \leqslant \int_{\mathbb{R}^{n}}\left|M_{\rho, \eta}\left(\left[b, \mathbb{I}_{\alpha}\right] f\right)(x)\right|^{q} d x \\
& \leqslant \int_{\mathbb{R}^{n}}\left|M_{\rho, \gamma}^{\sharp}\left(\left[b, \mathbb{I}_{\alpha}\right] f\right)(x)\right|^{q} d x+\sum_{k}\left|Q_{k}\right|\left(\frac{1}{\left|Q_{k}\right|} \int_{2 Q_{k}}\left|\left[b, \mathbb{I}_{\alpha}\right] f(x)\right| d x\right)^{q} \\
& \lesssim \int_{\mathbb{R}^{n}}\left|M_{\rho, \gamma}^{\sharp}\left(\left[b, \mathbb{I}_{\alpha}\right] f\right)(x)\right|^{q} d x+\sum_{k}\left|Q_{k}\right|\left(\inf _{y \in 2 Q_{k}} M_{\alpha+\beta, s}(f)(y)\right)^{q} \\
& \lesssim\left([b]_{\beta}^{\theta}\right)^{q} \int_{\mathbb{R}^{n}}\left|M_{\alpha+\beta, s}(f)(x)+M_{\beta, s}\left(\mathbb{I}_{\alpha} f\right)(x)\right|^{q} d x+\left([b]_{\beta}^{\theta}\right)^{q} \sum_{k} \int_{2 Q_{k}}\left|M_{\alpha+\beta, s}(f)(x)\right|^{q} d x \\
& \left.\left.\lesssim\left([b]_{\beta}^{\theta}\right)^{q}\left(\int_{\mathbb{R}^{n}}\left|M_{\alpha+\beta, s}(f)(x)\right|^{q} d x+\int_{\mathbb{R}^{n}} \mid M_{\beta, s}\left(\mathbb{I}_{\alpha} f\right)(x)\right)\right|^{q} d x\right) \\
& \lesssim\left([b]_{\beta}^{\theta}\right)^{q}\|f\|_{L^{p}\left(\mathbb{R}^{n}\right)^{\prime}}^{q}
\end{aligned}
$$

where we have used the finite overlapping property given by Proposition 2.3.

\section{Proofs of Theorems 1.5 and 1.6}

Let us first prove Theorem 1.5.

Choosing $\tau>1$, we only need to show that for any $\mathrm{H}_{\mathcal{L}}^{\mathrm{p}, \tau}$-atom $a$,

$$
\left\|\left[b, \mathbb{I}_{\alpha}\right] a\right\|_{L^{q}\left(\mathbb{R}^{n}\right)} \leqslant C
$$

holds, where $C$ is a constant independent of a. Suppose supp a $\subset B=B\left(x_{0}, r\right)$ with $r<\rho\left(x_{0}\right)$. Then

$$
\left\|\left[b, \mathbb{I}_{\alpha}\right] a\right\|_{L^{q}\left(\mathbb{R}^{n}\right)} \leqslant\left(\int_{2 B}\left|\left[b, \mathbb{I}_{\alpha}\right] a(x)\right|^{q} d x\right)^{1 / q}+\left(\int_{(2 B) c}\left|\left[b, \mathbb{I}_{\alpha}\right] a(x)\right|^{q} d x\right)^{1 / q}=A_{1}+A_{2} .
$$

Let $1 / q_{1}=1 / \tau-(\alpha+\beta) / n$. By Theorem 1.4 and the size condition of atom $a$, we have

$$
A_{1} \leqslant\left(\int_{2 B}\left|\left[b, \mathbb{I}_{\alpha}\right] a(x)\right|^{q_{1}} d x\right)^{1 / q_{1}}(2 r)^{\frac{n}{q}-\frac{n}{q_{1}}} \leqslant C\left(\int_{2 B}|a(x)|^{\tau} d x\right)^{1 / \tau}(2 r)^{\frac{n}{q}-\frac{n}{q_{1}}} \leqslant C(2 r)^{\frac{n}{\tau}-\frac{n}{p}}(2 r)^{\frac{n}{q}-\frac{n}{q_{1}}}=C .
$$


For $A_{2}$, we consider two cases, that are $r<\rho\left(x_{0}\right) / 4$ and $\rho\left(x_{0}\right) / 4 \leqslant r<\rho\left(x_{0}\right)$.

Case I: When $r<\rho\left(x_{0}\right) / 4$, by the vanishing condition of $a$, we have

$$
\left|\left[b, \mathbb{I}_{\alpha}\right] a(x)\right| \leqslant\left|b(x)-b_{B}\right| \int_{B}\left|K_{\alpha}(x, y)-K_{\alpha}\left(x, x_{0}\right)\right||a(y)| d y+\int_{B}\left|K_{\alpha}(x, y)\left(b(y)-b_{B}\right) a(y)\right| d y=A_{21}+A_{22} .
$$

Note that

$$
\int_{B}|a(y)| d y \lesssim r^{n-\frac{n}{p}}
$$

and

$$
\frac{1}{\left|2^{k} B\right|} \int_{2^{k} B}\left|b(x)-b_{B}\right|^{q} d x \lesssim\left([b]_{\beta}^{\theta}\right)^{q}\left(2^{k} r\right)^{\beta q}\left(1+\frac{2^{k} r}{\rho\left(x_{0}\right)}\right)^{\theta^{\prime} q} .
$$

When $x \in 2^{k+1} \mathrm{~B}\left(x_{0}, r\right) \backslash 2^{k} \mathrm{~B}\left(x_{0}, r\right)$, and $y \in B$, by Lemmas 2.7 and 2.2, we can take $\delta$ such that $0<\beta<$ $\delta<\delta^{\prime}$ and

$$
\left|K_{\alpha}(x, y)-K_{\alpha}\left(x, x_{0}\right)\right| \lesssim \frac{1}{\left(1+\frac{2^{k} r}{\rho\left(x_{0}\right)}\right)^{N /\left(k_{0}+1\right)}} \frac{r^{\delta}}{\left(2^{k} r\right)^{n+\delta-\alpha}}
$$

Noticing $1 / q=1 / p-(\alpha+\beta) / n$ and $p>\frac{n}{n+\beta}>\frac{n}{n+\delta}$, then we get

$$
\begin{aligned}
\int_{(2 B)^{c}}\left(A_{21}\right)^{q} d x & \lesssim r^{\left(n-\frac{n}{p}\right) q}\left([b]_{\beta}^{\Theta}\right)^{q} \sum_{k \geqslant 1} \frac{1}{\left(1+\frac{2^{k} r}{\rho\left(x_{0}\right)}\right)^{N q /\left(k_{0}+1\right)}} \frac{r^{\delta q}}{\left(2^{k} r\right)^{(n+\delta-\alpha) q}} \int_{2^{k} B}\left|b(x)-b_{B}\right|^{q} d x \\
& \lesssim\left([b]_{\beta}^{\Theta}\right)^{q} \sum_{k \geqslant 1} \frac{1}{\left(1+\frac{2^{k} r}{\rho\left(x_{0}\right)}\right)^{N q /\left(k_{0}+1\right)-\theta^{\prime} q}} 2^{k q\left(\frac{n}{p}-n-\delta\right)} \\
& \lesssim\left([b]_{\beta}^{\Theta}\right)^{q} \sum_{k \geqslant 1} 2^{k q\left(\frac{n}{p}-n-\delta\right)} \\
& \lesssim\left([b]_{\beta}^{\Theta}\right)^{q}
\end{aligned}
$$

For $x \in(2 B)^{c}, y \in B$, we have $|x-y| \approx\left|x-x_{0}\right|$. By Lemmas 2.7 and 2.2,

$$
\begin{aligned}
\left(\int_{2^{k+1} B \backslash 2^{k} B}\left|K_{\alpha}(x, y)\right|^{q} d x\right)^{1 / q} & \lesssim \frac{r^{\delta}}{\left(1+\frac{2^{k} r}{\rho\left(x_{0}\right)}\right)^{\frac{N}{k_{0}+1}}}\left(\int_{2^{k+1} B \backslash 2^{k} B} \frac{d x}{\left|x-x_{0}\right|^{q(n+\delta-\alpha)}}\right)^{1 / q} \\
& \lesssim \frac{2^{-k \delta}}{\left(1+\frac{2^{k} r}{\rho\left(x_{0}\right)}\right)^{\frac{N}{k_{0}+1}}} \frac{1}{\left(2^{k} r\right)^{\frac{n}{q^{\prime}}-\alpha}} .
\end{aligned}
$$

By Hölder's inequality and Lemma 2.5 we get

$$
\begin{aligned}
\int_{B}\left|b(y)-b_{B} \| a(y)\right| d y & \leqslant\left(\int_{B}|a(y)|^{\tau} d y\right)^{1 / \tau}\left(\int_{B}\left|b(y)-b_{B}\right|^{\tau^{\prime}} d y\right)^{1 / \tau^{\prime}} \\
& \lesssim[b]_{\beta}^{\theta} r^{\frac{n}{\tau}-\frac{n}{p}} r^{\beta+\frac{n}{\tau^{\prime}}}\left(1+\frac{r}{\rho\left(x_{0}\right)}\right)^{\theta^{\prime}} \\
& \lesssim[b]_{\beta}^{\theta} r^{n-\frac{n}{p}+\beta}\left(1+\frac{r}{\rho\left(x_{0}\right)}\right)^{\theta^{\prime}}
\end{aligned}
$$


Then, by Minkowski's inequality we get

$$
\begin{aligned}
\left(\int_{(2 B) c}\left(A_{22}\right)^{q} d x\right)^{1 / q} & \lesssim \int_{B}\left|b(y)-b_{B} \| a(y)\right| d y\left(\sum_{k \geqslant 1} \int_{2^{k+1} B \backslash 2^{k_{B}} B}\left|K_{\alpha}(x, y)\right|^{q} d x\right)^{1 / q} \\
& \lesssim[b]_{\beta}^{\theta} r^{n-\frac{n}{p}+\beta}\left(1+\frac{r}{\rho\left(x_{0}\right)}\right)^{\theta^{\prime}} \sum_{k \geqslant 1} \frac{2^{-k \delta}}{\left(1+\frac{2^{k} r}{\rho\left(x_{0}\right)}\right)^{\frac{N}{k_{0}+1}}} \frac{1}{\left(2^{k} r\right)^{\frac{n}{q^{\prime}}-\alpha}} \\
& \lesssim[b]_{\beta}^{\theta} \sum_{k \geqslant 1} \frac{1}{2^{k\left(n-\frac{n}{p}+\beta+\delta\right)}} \\
& \lesssim[b]_{\beta}^{\theta} .
\end{aligned}
$$

Case II: When $\rho\left(x_{0}\right) / 4 \leqslant r<\rho\left(x_{0}\right)$, this is $\frac{r}{\rho\left(x_{0}\right)} \geqslant 1 / 4$. The atom a does not satisfy the vanishing condition. By Minkowski's inequality,

$$
\begin{aligned}
A_{2} \leqslant & \left\{\int_{(2 B)^{c}}\left|b(x)-b_{B}\right|^{q}\left|\int_{B} K_{\alpha}(x, y) a(y) d y\right|^{q} d x\right\}^{1 / q} \\
& +\left\{\int_{(2 B)^{c}}\left|\int_{B}\right| K_{\alpha}(x, y)\left(b(y)-b_{B}\right) a(y)|d y|^{q} d x\right\}^{1 / q} \\
= & A_{21}^{\prime}+A_{22}^{\prime} .
\end{aligned}
$$

When $y \in B, x \in 2^{k+1} B \backslash 2^{k} B$, we have

$$
\begin{aligned}
\left|K_{\alpha}(x, y)\right| & \lesssim \frac{1}{\left(1+\frac{2^{k} r}{\rho\left(x_{0}\right)}\right)^{\frac{N}{k_{0}+1}}} \frac{1}{\left(2^{k} r\right)^{n-\alpha}}, \\
\int_{B}|a(y)| d y & \lesssim r^{n-\frac{n}{p}}
\end{aligned}
$$

and

$$
\int_{2^{k} B}\left|b(x)-b_{B}\right|^{q} d x \lesssim\left([b]_{\beta}^{\theta}\right)^{q}\left(2^{k} r\right)^{n+\beta q}\left(1+\frac{2^{k} r}{\rho\left(x_{0}\right)}\right)^{\theta^{\prime} q} .
$$

Note that $\frac{r}{\rho\left(x_{0}\right)} \geqslant 1 / 4$, then

$$
\begin{aligned}
& \left(A_{21}^{\prime}\right)^{q} \lesssim\left([b]_{\beta}^{\theta}\right)^{q} \sum_{k \geqslant 1} \frac{1}{\left(1+\frac{2^{k} r}{\rho\left(x_{0}\right)}\right)^{\frac{q N}{k_{0}+1}-q \theta^{\prime}}} \frac{\left(2^{k} r\right)^{n+\beta q}}{\left(2^{k} r\right)^{(n-\alpha) q}} r^{\left(n-\frac{n}{p}\right) q} \\
& \lesssim\left([b]_{\beta}^{\Theta}\right)^{q} \sum_{k \geqslant 1} \frac{1}{\left(2^{k}\right)^{\frac{q N}{k_{0}+1}-q \theta^{\prime}}}\left(2^{k}\right)^{\left(\frac{n}{p}-n\right) q} \lesssim\left([b]_{\beta}^{\theta}\right)^{q} .
\end{aligned}
$$

Since $\mathrm{N}$ can be chosen large enough, the last series converges.

The estimate of $A_{22}^{\prime}$ is exactly the same as $\left\|A_{22}\right\|_{L_{(}\left((2 B)^{c}\right)}$, we omit the detail of the proof. Then the proof of Theorem 1.5 is finished.

Finally, we proceed to prove Theorem 1.6.

Let $f \in H_{\mathcal{L}}^{\frac{n}{n+\beta}}\left(\mathbb{R}^{n}\right)$, we write $f=\sum_{j=-\infty}^{\infty} \lambda_{j} a_{j}$, where each $a_{j}$ is an $H_{\mathcal{L}}^{\frac{n}{n+\beta}}, l$-atom, $1<l<\frac{n}{\alpha+\beta}$, and

$$
\left(\sum_{j=-\infty}^{\infty}\left|\lambda_{j}\right|^{\frac{n}{n+\beta}}\right)^{\frac{n+\beta}{n}} \leqslant 2\|f\|_{H_{\mathcal{L}}^{\frac{n}{n+\beta}}\left(\mathbb{R}^{n}\right)}
$$


Suppose that suppa $a_{j} \subset B_{j}=B\left(x_{j}, r_{j}\right)$ with $r_{j}<\rho\left(x_{j}\right)$. Write

$$
\begin{aligned}
{\left[b, \mathbb{I}_{\alpha}\right] f(x)=} & \sum_{j=-\infty}^{\infty} \lambda_{j}\left[b, \mathbb{I}_{\alpha}\right] a_{j}(x) \chi_{8 B_{j}}(x)+\sum_{j: r_{j} \geqslant \rho\left(x_{j}\right) / 4} \lambda_{j}\left(b(x)-b_{B_{j}}\right) \mathbb{I}_{\alpha} a_{j}(x) \chi_{\left(8 B_{j}\right) c}(x) \\
& +\sum_{j: r_{j}<\rho\left(x_{j}\right) / 4} \lambda_{j}\left(b(x)-b_{B_{j}}\right) \mathbb{I}_{\alpha} a_{j}(x) \chi_{\left(8 B_{j}\right) c}(x)-\sum_{j=-\infty}^{\infty} \lambda_{j} \mathbb{I}_{\alpha}\left(\left(b-b_{B_{j}}\right) a_{j}\right)(x) \chi_{\left(8 B_{j}\right) c}(x) \\
= & \sum_{i=1}^{4} \sum_{j=-\infty}^{\infty} \lambda_{j} A_{i j}(x) .
\end{aligned}
$$

In the following, we always let $q=\frac{n}{n-\alpha}$. Note that

$$
\left(\int_{B_{j}}\left|a_{j}(x)\right|^{l} d x\right)^{1 / l} \lesssim\left|B_{j}\right|^{\frac{1}{l}-\frac{n+\beta}{n}} .
$$

Choose $t>\frac{n-\alpha}{n-\alpha-\beta}$ such that $\frac{1}{q t}=\frac{1}{l}-\frac{\alpha+\beta}{n}$. By Hölder's inequality and Theorem 1.4 we get

$$
\begin{aligned}
\left\|A_{1, j}\right\|_{L q\left(\mathbb{R}^{n}\right)} & \lesssim\left(\int_{8 B_{j}}\left|\left[b, \mathbb{I}_{\alpha}\right] a_{j}(x)\right|^{q t} d x\right)^{\frac{1}{q t}} r_{j}^{\frac{n}{q t^{\prime}}} \\
& \lesssim[b]_{\beta}^{\theta} r_{j}^{\frac{n}{q t^{\prime}}}\left(\int_{B_{j}}\left|a_{j}(x)\right|^{l} d x\right)^{1 / l} \lesssim[b]_{\beta}^{\Theta}\left|B_{j}\right|^{\frac{1}{q t^{\prime}}+\frac{1}{l}-\frac{n+\beta}{n}} \lesssim[b]_{\beta}^{\theta} .
\end{aligned}
$$

Noticing $0<\frac{n}{n+\beta}<1$, we get

$$
\begin{gathered}
\left\|\sum_{j=-\infty}^{\infty} \lambda_{j} A_{1 j}\right\|_{L^{q}\left(\mathbb{R}^{n}\right)} \lesssim \sum_{j=-\infty}^{\infty}\left|\lambda_{j}\right|\left\|A_{1 j}\right\|_{L^{q}\left(\mathbb{R}^{n}\right)} \lesssim[b]_{\beta}^{\theta} \sum_{j=-\infty}^{\infty}\left|\lambda_{j}\right| \lesssim[b]_{\beta}^{\theta}\left(\sum_{j=-\infty}^{\infty}\left|\lambda_{j}\right|^{\frac{n}{n+\beta}}\right)^{\frac{n+\beta}{n}} \\
\lesssim[b]_{\beta}^{\theta}\|f\|_{H_{\mathcal{L}}^{\frac{n}{n+\beta}}\left(\mathbb{R}^{n}\right)}
\end{gathered}
$$

Then

$$
\left|\left\{x \in \mathbb{R}^{n}:\left|\sum_{j=-\infty}^{\infty} \lambda_{j} A_{1 j}\right|>\frac{\lambda}{4}\right\}\right| \lesssim \frac{\left([b]_{\beta}^{\Theta}\right)^{q}}{\lambda^{q}}\|f\|_{H_{\mathcal{L}}^{\mathrm{q}+\beta}\left(\mathbb{R}^{n}\right)}^{q} .
$$

Since $x \in B_{j}, y \in 2^{k+1} B_{j} \backslash 2^{k} B_{j}$, we have $|x-y| \approx\left|x-x_{j}\right| \approx 2^{k} r_{j}$, and by Lemma 2.2 we get

$$
\frac{1}{\left(1+\frac{|x-y|}{\rho(x)}\right)^{N}} \lesssim \frac{1}{\left(1+\frac{2^{\mathrm{k}} r_{j}}{\rho\left(x_{j}\right)}\right)^{\frac{N}{\mathrm{~K}_{0}+1}}} .
$$

Note that $\int_{B_{j}}\left|a_{j}(y)\right| d y \leqslant r_{j}^{-\beta}$, and $r_{j} / \rho\left(x_{j}\right) \geqslant 1 / 4$. Then

$$
\begin{aligned}
\left\|A_{2, j}(x)\right\|_{L^{q}\left(\mathbb{R}^{n}\right)}^{q} & =\sum_{k \geqslant 3} \int_{2^{k+1} B_{j} \backslash 2^{k} B_{j}}\left|b(x)-b_{B_{j}}\right|^{q}\left(\int_{B_{j}} \frac{1}{\left(1+\frac{|x-y|}{\rho(x)}\right)^{N}} \frac{1}{|x-y|^{n-\alpha}}\left|a_{j}(y)\right| d y\right)^{q} d x \\
& \lesssim \sum_{k \geqslant 3} \frac{1}{\left(1+\frac{2^{k} r_{j}}{\rho\left(x_{j}\right)}\right)^{\frac{N q}{k_{0}+1}}} \frac{1}{\left(2^{k} r_{j}\right)^{(n-\alpha) q}} \int_{2^{k+1} B_{j}} \mid b(x)-b_{B_{j}}{ }^{q} d x\left(\int_{B_{j}}\left|a_{j}(y)\right| d y\right)^{q}
\end{aligned}
$$




$$
\begin{aligned}
& \lesssim\left([b]_{\beta}^{\Theta}\right)^{q} \sum_{k \geqslant 3} \frac{1}{\left(1+\frac{2^{k} r_{j}}{\rho\left(x_{j}\right)}\right)\left(\frac{N}{k_{0}+1}-\theta^{\prime}\right) q}\left(2^{k} B_{j}\right)^{\beta q} r_{j}^{-\beta q} \\
& \lesssim\left([b]_{\beta}^{\theta}\right)^{q} \sum_{k \geqslant 1} \frac{1}{2^{k q\left(\frac{N}{k_{0}+1}-\theta^{\prime}-\beta\right)}} \\
& \lesssim\left([b]_{\beta}^{\Theta}\right)^{q} .
\end{aligned}
$$

Then

$$
\left\|\sum_{j=-\infty}^{\infty} \lambda_{j} A_{2 j}\right\|_{L^{q}\left(\mathbb{R}^{n}\right)} \lesssim[b]_{\beta}^{\Theta}\|f\|_{H_{\mathcal{L}}^{\frac{n}{n+\beta}}\left(\mathbb{R}^{n}\right)} .
$$

Therefore

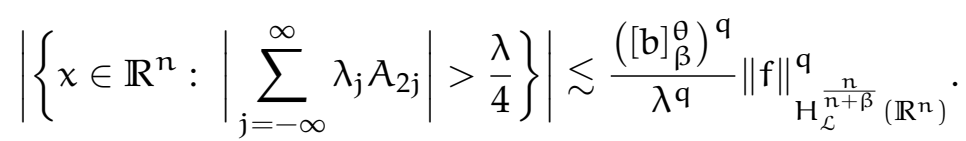

When $x \in 2^{k+1} B_{j} \backslash 2^{k} B_{j}$, and $y \in B_{j}$, by Lemmas 2.7 and 2.2 we have

$$
\left|K_{\alpha}(x, y)-K_{\alpha}\left(x, x_{j}\right)\right| \lesssim \frac{1}{\left(1+\frac{2^{k} r_{j}}{\rho\left(x_{j}\right)}\right)^{N /\left(k_{0}+1\right)}} \frac{r_{j}^{\delta}}{\left(2^{k} r_{j}\right)^{n+\delta-\alpha}}
$$

Thus, by the vanishing condition of $a_{j}$ and $0<\beta<\delta<\delta^{\prime}$ we have

$$
\begin{aligned}
\left\|A_{3, j}(x)\right\|_{L^{q}\left(\mathbb{R}^{n}\right)}^{q} & =\sum_{k \geqslant 3} \int_{2^{k+1} B_{j} \backslash 2^{k} B_{j}}\left|b(x)-b_{B_{j}}\right|^{q}\left(\int_{B_{j}}\left|K_{\alpha}(x, y)-K_{\alpha}\left(x, x_{j}\right) \| a_{j}(y)\right| d y\right)^{q} d x \\
& \lesssim \sum_{k \geqslant 3} \frac{1}{\left(1+\frac{2^{k} r_{j}}{\rho\left(x_{j}\right)}\right)^{\frac{N q}{k_{0}+1}}} \frac{r_{j}^{\delta q}}{\left(2^{k} r_{j}\right)^{(n+\delta-\alpha) q}} \int_{2^{k+1} B_{j}}\left|b(x)-b_{B_{j}}\right|^{q} d x\left(\int_{B_{j}}\left|a_{j}(y)\right| d y\right)^{q} \\
& \left.\lesssim\left([b]_{\beta}^{\theta}\right)^{q} \sum_{k \geqslant 3} \frac{1}{\left(1+\frac{2^{k} r_{j}}{\rho\left(x_{j}\right)}\right)}\right)^{\left(\frac{N}{k_{0}+1}-\theta^{\prime}\right) q} \frac{r_{j}^{\delta q}}{\left(2^{k} r_{j}\right)^{(n+\delta-\alpha) q}}\left(2^{k} r_{j}\right)^{n+\beta q} r_{j}^{-\beta q} \\
& \lesssim\left([b]_{\beta}^{\Theta}\right)^{q} \sum_{k \geqslant 1} \frac{1}{2^{k(\delta-\beta)}} \\
& \lesssim\left([b]_{\beta}^{\Theta}\right)^{q} .
\end{aligned}
$$

Then

$$
\left\|\sum_{j=-\infty}^{\infty} \lambda_{j} A_{3 j}\right\|_{L^{q}\left(\mathbb{R}^{n}\right)} \lesssim[b]_{\beta}^{\Theta}\|f\|_{H_{\mathcal{L}}^{\frac{n}{n+\beta}}\left(\mathbb{R}^{n}\right)} .
$$

Therefore

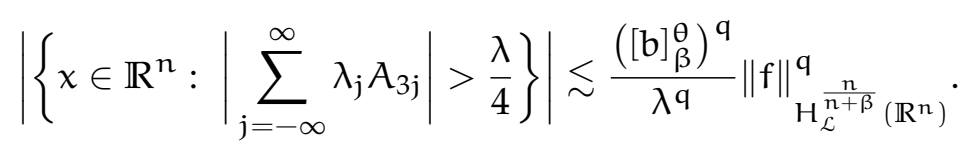

Note that

$$
\left\|\left(b-b_{B_{j}}\right) a_{j}\right\|_{L^{1}} \leqslant\left(\int_{B_{j}}\left|b(x)-b_{B_{j}}\right|^{l^{\prime}} d x\right)^{1 / l^{\prime}}\left(\int_{B_{j}}\left|a_{j}(x)\right|^{l} d x\right)^{1 / l}
$$




$$
\lesssim[b]_{\beta}^{\theta} r_{j}^{\frac{n}{l}-n-\beta+\frac{n}{l^{\prime}}+\beta}\left(1+\frac{r_{j}}{\rho\left(x_{j}\right)}\right)^{\theta^{\prime}} \lesssim[b]_{\beta}^{\theta}
$$

and

$$
\left|A_{4 j}(x)\right| \leqslant \sum_{j=-\infty}^{\infty}\left|\lambda_{j}\right| \mathbb{I}_{\alpha}\left(\left|\left(b-b_{B_{j}}\right) a_{j}\right|\right)(x) \chi_{\left(8 B_{j}\right) c}(x) \leqslant \mathbb{I}_{\alpha}\left(\sum_{j=-\infty}^{\infty}\left|\lambda_{j}\left(b-b_{B_{j}}\right) a_{j}\right|\right)(x) .
$$

By the boundedness of $\mathbb{I}_{\alpha}$ from $L^{1}\left(\mathbb{R}^{n}\right)$ to $W L^{q}\left(\mathbb{R}^{n}\right)$ (see Corollary 2.8) we get

$$
\begin{aligned}
& \left|\left\{x \in \mathbb{R}^{n}:\left|\sum_{j=-\infty}^{\infty} \lambda_{j} A_{4 j}\right|>\frac{\lambda}{4}\right\}\right| \leqslant\left|\left\{x \in \mathbb{R}^{n}:\left|\mathbb{I}_{\alpha}\left(\sum_{j=-\infty}^{\infty}\left|\lambda_{j}\left(b-b_{B_{j}}\right) a_{j}\right|\right)(x)\right|>\frac{\lambda}{4}\right\}\right| \\
& \lesssim \frac{1}{\lambda^{q}}\left\|\sum_{j=-\infty}^{\infty}\left|\lambda_{j}\left(b-b_{B_{j}}\right) a_{j}\right|\right\|_{L^{1}\left(\mathbb{R}^{n}\right)}^{q} \\
& \lesssim \frac{1}{\lambda^{q}}\left(\sum_{j=-\infty}^{\infty}\left|\lambda_{j}\right|\left\|\left(b-b_{B_{j}}\right) a_{j}\right\|_{L^{1}\left(\mathbb{R}^{n}\right)}\right)^{q} \\
& \lesssim \frac{\left([b]_{\beta}^{\Theta}\right)^{q}}{\lambda^{q}}\left(\sum_{j=-\infty}^{\infty}\left|\lambda_{j}\right|\right)^{q} \lesssim \frac{\left([b]_{\beta}^{\Theta}\right)^{q}}{\lambda^{q}}\|f\|_{H_{\mathcal{L}}^{q+\beta}\left(\mathbb{R}^{n}\right)}^{q} .
\end{aligned}
$$

Thus,

$$
\begin{aligned}
& \left|\left\{x \in \mathbb{R}^{n}:\left|\sum_{i=1}^{4} \sum_{j=-\infty}^{\infty} \lambda_{j} A_{i j}\right|>\lambda\right\}\right| \lesssim \sum_{i=1}^{4}\left|\left\{x \in \mathbb{R}^{n}:\left|\sum_{j=-\infty}^{\infty} \lambda_{j} A_{i j}\right|>\frac{\lambda}{4}\right\}\right|
\end{aligned}
$$

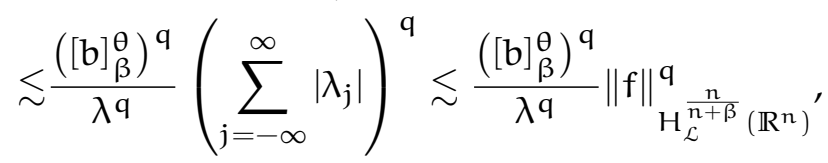

which completes the proof of Theorem 1.6.

\section{References}

[1] B. Bongioanni, E. Harboure, O. Salinas, Commutators of Riesz transforms related to Schrödinger operators, J. Fourier Anal. Appl., 17 (2011), 115-134.1, 2.4

[2] T. A. Bui, Weighted estimates for commutators of some singular integrals related to Schrödinger operators, Bull. Sci. Math., 138 (2014), 270-292.1, 2

[3] S. Chanillo, A note on commutator, Indiana Univ. Math. J., 31 (1982), 7-16.1

[4] J. Dziubański, J. Zienkiewicz, Hardy space $\mathrm{H}^{1}$ associated to Schrödinger operator with potential satisfying reverse Hölder inequality, Rev. Mat. Iberoamericana, 15 (1999), 279-296.1, 2.3

[5] J. Dziubański, J. Zienkiewicz, $\mathrm{H}^{\mathrm{p}}$ spaces associated with Schrödinger operators with potentials from reverse Hölder classes, Colloq. Math., 98 (2003), 5-38.1, 1.3, 2.6

[6] D. Goldberg, A local version of real Hardy spaces, Duke Math. J., 46 (1979), 27-42.1

[7] Y. Liu, J. Sheng, Some estimates for commutators of Riesz transforms associated with Schrödinger operators, J. Math. Anal. Appl., 419 (2014), 298-328.1, 2.5

[8] S. Z. Lu, Q. Wu, D.C. Yang, Boundedness of commutators on Hardy type spaces, Sci. China Ser. A, 45 (2002), $984-997.1$

[9] M. Paluszyński, Characterization of the Besov spaces via the commutator operator of Coifman, Rochberg and Weiss, Indiana Univ. Math. J., 44 (1995), 1-17. 1

[10] Z. W. Shen, $\mathrm{L}^{\mathfrak{P}}$ estimates for Schrödinger operators with certain potentials, Ann. Inst. Fourier, 45 (1995), 513-546.1, 2.1 